
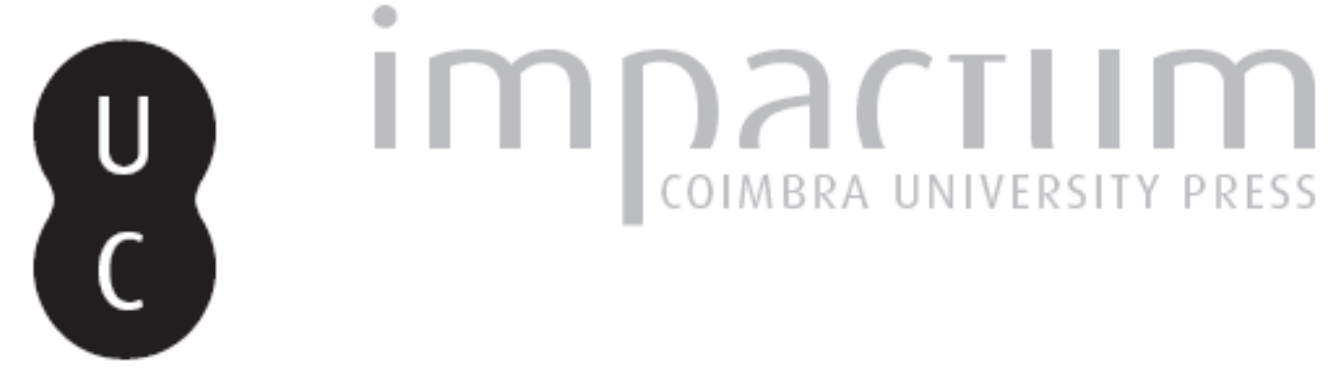

\title{
[Recensão a] José de Almada Negreiros, Prose d'avanguardia, a cura di Valeria Tocco; Nome di battaglia, trad. di Andrea Ragusa
}

Autor(es): Bettini, Clelia

Publicado por: Imprensa da Universidade de Coimbra

URL persistente:

URI:http://hdl.handle.net/10316.2/42400

DOI:

DOI:https://doi.org/10.14195/0870-8584_10_13

Accessed : $\quad$ 26-Apr-2023 13:57:31

A navegação consulta e descarregamento dos títulos inseridos nas Bibliotecas Digitais UC Digitalis, UC Pombalina e UC Impactum, pressupõem a aceitação plena e sem reservas dos Termos e Condições de Uso destas Bibliotecas Digitais, disponíveis em https://digitalis.uc.pt/pt-pt/termos.

Conforme exposto nos referidos Termos e Condições de Uso, o descarregamento de títulos de acesso restrito requer uma licença válida de autorização devendo o utilizador aceder ao(s) documento(s) a partir de um endereço de IP da instituição detentora da supramencionada licença.

Ao utilizador é apenas permitido o descarregamento para uso pessoal, pelo que o emprego do(s) título(s) descarregado(s) para outro fim, designadamente comercial, carece de autorização do respetivo autor ou editor da obra.

Na medida em que todas as obras da UC Digitalis se encontram protegidas pelo Código do Direito de Autor e Direitos Conexos e demais legislação aplicável, toda a cópia, parcial ou total, deste documento, nos casos em que é legalmente admitida, deverá conter ou fazer-se acompanhar por este aviso.

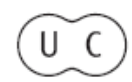




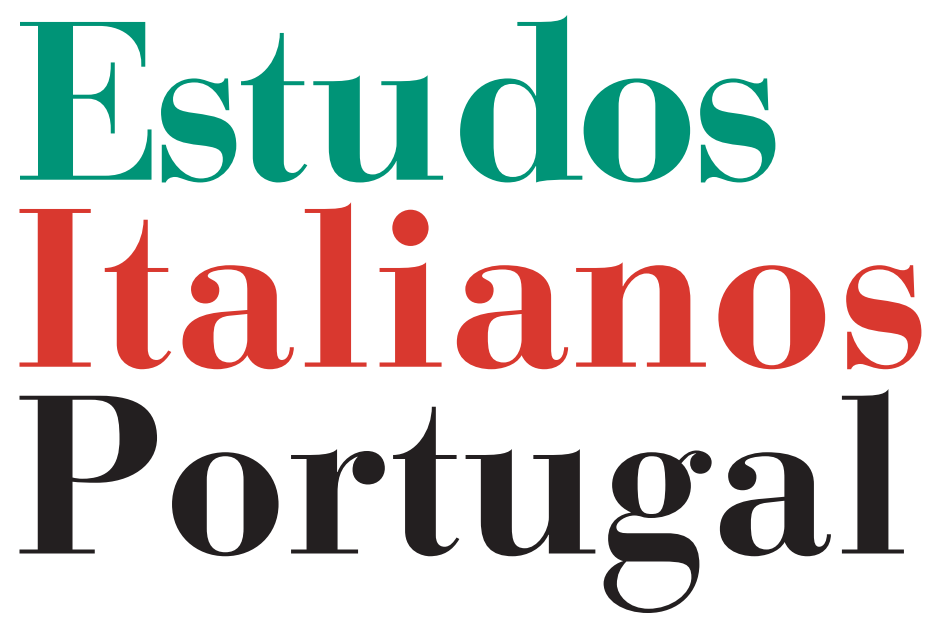

Instituto

Italiano

de Cultura

de Lisboa

Nova Série

$\mathrm{N}^{\mathbf{0}} 10$

2015 
agora foste vencido por uma donzela chorando!...”), e que a versão italiana acaba por não contemplar: "Bimarder arrestò il passo, spaventato; credeva che nessuno conoscesse ancora il suo nome." (p. 55)

\section{Coteje-se, por último:}

"Isto dizia ela, porque era costume mui guardado naquela terra, e ficara doutro tempo, sob grandes penas, proibido, não se pôr mulher nenhuma em cabelo senão por seu marido." (p. 57)

"Diceva questo perché strapparsi i capelli era un'usanza molto antica di quella terra, ma era proibito da molto tempo, con grandi pene, a ogni donna, a meno che non lo facesse per il proprio marito." (p. 39)

"Pôr-se em cabelo" (mostrar a cabeça nua, sem qualquer véu ou toucado) não é sinónimo de "strapparsi i capelli". Para evitar tais confusões, basta uma análise mais paciente e mais exigente $\mathrm{da}$ obra de Bernardim Ribeiro.

O leitor que comparar o texto original com a tradução de Alfonso Bruno Parisini notará discrepâncias como as que procurámos, a título de exemplo, assinalar. Não deixará de apreciar, porém, o trabalho realizado e o equilíbrio ami- úde conseguido. Cumpre louvar a coragem subjacente à preparação da Storia di una giovane fanciulla e agradecer o contributo dado à difusão de uma extraordinária obra portuguesa do tempo do Renascimento.

ISABEL ALMEIDA

José de Almada Negreiros, Prose d'avanguardia, a cura di Valeria Tocco, Perugia, Edizioni dell'Urogallo, 2014, pp. 191.

José de Almada Negreiros, Nome di battaglia, trad. di Andrea Ragusa, Perugia, Edizioni dell'Urogallo, 2014, pp. $184+$ n.n.

Nel passato 2014, un piccolo editore di Perugia, le Edizioni dell'Urogallo, ha dato alle stampe due volumi eleganti su cui spicca la firma dei uno dei più importanti protagonisti del Modernismo portoghese: José de Almada Negreiros. Entrambe le pubblicazioni sono il risultato del lavoro di un'équipe di studiosi e traduttori italiani, coordinata da Valeria Tocco (Università di Pisa), che ha portato alla realizzazione del congresso internazionale Almada Negreiros: un trait d'union tra arti e culture (Pisa 10-12 giugno 2015), e della mostra Almada Negreiros, artista prismati- 
co (Pisa, 10-27 giugno 2015) dedicata all'opera plastica dell'artista portoghese.

Il volume Prose d'avanguardia raccoglie i manifesti e le "poesie plastico-narrative" di Almada, queste ultime per la prima volta tradotte in lingue italiana, a cui seguono un interessante apparato di note al testo e tre saggi critici di Valeria Tocco, curatrice del volume. Nome di battaglia (Nome de guerra), invece, è l'unico romanzo pubblicato da Almada, scritto nel 1925 ma edito solamente nel 1938, che possiamo leggere ora nella traduzione italiana di Andrea Ragusa, corredata da una postfazione di Gustavo Rubim. Si tratta di due libri preziosi per i lettori italiani che non hanno familiarità con la lingua portoghese e che hanno ora la possibilità di approfondire la conoscenza dell'opera di Almada Negreiros, artista poliedrico e figura di spicco del modernismo portoghese, protagonista assieme a Fernando Pessoa dell'avventura letteraria catalizzata dai due numeri della rivista Orpheu.

José de Almada Negreiros è stato uno degli artisti portoghesi più significativi del secolo passato. L'Almada modernista di cui si occupano i due volumi editi da
Urogallo era in primo luogo un personaggio irriverente, un artista ironico, sarcastico e originale. Nemico dei "parrucconi", degli accademici pomposi, si dedicava con piacere a punzecchiare la sonnolenza compiacente di un paese che si dondolava dolcemente ai margini dell'Europa scossa dalle avanguardie e dalla Prima Guerra Mondiale. I testi raccolti nel volume $L e$ prose d'avanguardia sono stati tradotti dalla curatrice, Valeria Tocco, insieme a Mauro La Mancusa e Andrea Ragusa, con la partecipazione di alcuni allievi del Corso di Laurea Magistrale in Traduzione Letteraria e Saggistica dell'Università di Pisa, nell'ambito di un laboratorio di traduzione. Presenta una selezione di testi "compromessi, in un modo o nell'altro, con i principi dell'avanguardia storica", nella misura in cui si tratta di manifesti programmatici e di prose che "più caratterizzano l'originale rielaborazione e superamento della prassi futurista compiuta da Almada" (p. 175). Si tratta di testi pubblicati tra il 1915 e il 1921 , in piena fase modernista dunque, ma che sono a tutti gli effetti fondamentali per la comprensione anche della produzione artistica successiva, non solo di Almada Negreiros, ma più 
in generale della poesia e della narrativa portoghese del Novecento.

Manifesto anti-Dantas e per esteso, primo testo della raccolta, costituisce l'esordio letterario di Almada. Ha la forma di un manifesto di matrice marinettiana, in cui il poeta, poco più che ventenne, si scaglia contro il celebre drammaturgo Júlio Dantas, simbolo di un modo "passatista" di intendere la vita e l'arte. Sin da questo primo pamphlet Almada dichiara esplicitamente la propria affinità con le avanguardie e, in particolare, con il futurismo di Marinetti, firmandosi Poeta di Orpheu - Futurista e Tutto. In quegli anni collabora alla rivista Orpheu, centro propulsore e catalizzatore dei modernismi portoghesi e pubblica sul numero unico Portugal Futurista - Publicação eventual, l'Ultimatum Futurista alle generazioni portoghesi del secolo $X X$, così come le prose Saltimbanchi e I balletti russi a Lisbona, tutti presenti nell'antologia pubblicata da Urogallo.

Il manifesto dal titolo $I$ balletti russi a Lisbona rivela un aspetto originale della variegata produzione artistica di Almada Negreiros, quello di coreografo e ballerino di avanguardia. Come molti artisti europei, restò profondamente im- pressionato dai Ballets Russes di Sergej Djagilev, a cui assistette a Lisbona nel dicembre del 1917, che lo spinsero a scrivere una delle prose più decise contro una diffusa forma di impasse culturale, timorosa e provinciale, che prostrava in modo a suo vedere drammatico il popolo portoghese. La transcodificazione futurista dei Ballets Russes di Djagilev esplode nel ricorso ossessivo allo stampatello e nell'attacco continuo rivolto alla miseria artistica in cui il popolo portoghese vive immerso: $\mathrm{i}$ Ballets Russes rappresentano per Almada una chance da non perdere, un incontro necessario con l'Arte capace di educare alla disciplina e un indispensabile contatto con il "bello e luminoso cervello dell'Europa", capace di rischiarare l'oscurantismo lusitano.

Il testo intitolato Esposizione Amadeo de Souza-Cardoso Lega Navale di Lisbona, scritto in occasione della mostra personale del pittore portoghese a Lisbona, costituisce invece uno dei primi esempi di riflessione sull'arte contemporanea pubblicati da Almada Negreiros. Amadeo de Souza-Cardoso era vicino ad Amedeo Modigliani, che conobbe e frequentò a Parigi, e Almada lo definisce come "la prima Scoperta del Portogallo 
nell'Europa del XX secolo" (p. 24), entrando in aperta polemica con la onnipresente retorica nazionale dei descobrimentos, sotto cui si celavano un'ignoranza e un'indifferenza, e che, purtroppo, avrebbero continuato ad asfissiare il mondo culturale portoghese ancora per molti decenni.

Invece, nelle quattro "novelle plastiche" raccolte nel volume di cui scriviamo (K4, La stiratrice, Saltimbanchi e L'invenzione del giorno chiaro, 1915-1921) l'importanza della pittura, in particolare del colore, si rivela già molto evidente, come spiega Valeria Tocco nel saggio che dedica loro, dal titolo Il visibile narrare (pp. 184187). Basta pensare ai soprannomi cromatici del famoso Clube dos 5 di cui facevano parte Almada e altri artisti suoi amici, o alla teoria dei colori di Kandinsky, omaggiato dalla $K$ del titolo di $K 4$, proseguendo poi con il simultaneismo di Sonia e Robert Delaunay evocato invece dal sottotitolo di Saltimbanchi - Contrasti simultanei. Inoltre, alcune delle scene descritte plasticamente in queste quattro prose si ritrovano effettivamente dipinte in alcuni dei quadri e dei pannelli a olio realizzati da Almada alcuni anni più tardi, come segnala la curatrice del volume (p. 187).

La prosa conclusiva, L'invenzione del giorno chiaro, per la storia che porta con sé, merita forse un'attenzione particolare. Si tratta del testo, rivisto e aumentato, di una conferenza proferita da Almada alla Lega Navale di Lisbona il 3 marzo del 1921, e fu il primo titolo pubblicato dal recentissimo "progetto editoriale" Olisipo di Fernando Pessoa, nel dicembre dello stesso anno. Il piano editoriale della Olisipo pessoana aveva il proposito di colmare quelle che, secondo il poeta, erano le principali lacune del mercato editoriale portoghese (e brasiliano, come ripete quasi ossessivamente) dell'epoca: la scelta dei titoli e degli autori, che dovevano essere fra i più significativi del panorama culturale mondiale, dare preferenza a opere non eccessivamente estese e pubblicare edizioni economiche. Alla luce di questa connessione pessoana, L'invenzione del giorno chiaro assume il valore di una sorta di presentazione del nuovo projecto editorial (che pubblicherà solamente quattro volumi in tutto), e non è un caso che si apra, nell'edizione a stampa, con un frammento intitolato proprio $I l$ libro, assente dalla versione decla- 
mata da Almada alla Lega Navale. Almada immagina di entrare in una libreria e metabolizza le critiche e le intenzioni espresse da Fernando Pessoa in merito alla futura Olisipo, trasformandole in osservazioni di una realtà immaginata ma plausibile. Sebbene siano trascorsi quasi 100 anni dalla pubblicazione del primo titolo della Olisipo, le idee pessoane sull'editoria e le provocazioni in "prosa-plastica" di Almada che ne derivarono, risultano non solo molto attuali, ma anche molto utili, per chi abbia ancora voglia di riflettere sull'importanza dell'editoria e sulle scelte che un'editoria di qualità deve compiere, ancora oggi, in Italia come in Portogallo.

Nome di battaglia, come si è detto in apertura, è l'unico romanzo scritto da Almada Negreiros. Lo si può definire un romanzo, sebbene tale definizione stia saltando irrimediabilmente in aria, in Europa, proprio negli anni in cui Almada lo scrive. In ogni caso, il testo presenta un filo narrativo, certo frammentario, una serie di personaggi principali, affiancati da altri secondari, un'ambientazione cittadina (Lisbona e i suoi dintorni), ma senza abbondanza di particolari. In Nome di batta- glia, Almada racconta il percorso di formazione di Luís Antunes, un giovanotto di provincia che arriva nella capitale portoghese in cerca di avventura. È iniziato ai piaceri della vita cittadina dallo zio, un uomo chiamato con l'epiteto D. Jorge, esperto conoscitore della Lisbona notturna. È un romanzo fatto di ricordi, descrizioni, divagazioni e riflessioni che si mescolano tra una stanza d'albergo e il night-club dove Antunes trascorre il proprio tempo, in un alternarsi confuso di notte e giorno. È un testo complesso e volutamente enigmatico, che si presta a vari livelli di lettura: è, probabilmente, un auto-ritratto in forma di romanzo dello stesso Almada, ma è soprattutto una riflessione sull'identità $\mathrm{e}$ ha l'incedere pausato e coraggioso di una meditazione metafisica sulla verità. Che rapporto esiste tra gli individui, la realtà e $\mathrm{i}$ nomi che usiamo per nominarla? È uno dei dibattiti più antichi della filosofia occidentale (già Gorgia, Platone e Aristotele hanno sollevato questioni fondamentali in merito), ma che si ripresenta, carico di inquietudine, agli inizi del secolo passato. Almada racconta una possibile ricerca dell'anonimato, l'uso di un nome de guerra che liberi dalla 
costrizione dell'esistenza predeterminata dal nome e cognome, e dall'albero genealogico, un mistero inconoscibile che tutti, volenti o nolenti, portiamo dentro noi stessi. È questo "l'aspetto antinaturalista di Nome di battaglia, la sua sfumatura anti-Zola", come osserva Gustavo Rubim nella postfazione al romanzo (p. 181), che lo distanzia anni luce dalla pratica corrente del romanzo portoghese, non solo coevo, ma anche successivo: pochi anni dopo, infatti, cominceranno gli anni forti del neo-realismo, con un'idea di letteratura radicalmente diversa, per intenti e per modalità narrative, da quella pratica da Almada.

Tuttavia, il romanzo di Almada è, a conti fatti, una lunga riflessione sul vivere e le sfide che esso comporta, intessuta con i fili della decostruzione ironica propria del modernismo. Antunes è in lotta con la realtà e le sue convenzioni, con tutto ciò che il mondo circostante si aspetta da uomo della sua età, della sua estrazione sociale, che porta il suo nome. Nella consapevolezza rischiarata dalla notte piena di stelle, che rimanda alla prosa Linvenzione del giorno chiaro, comprende che la vera risposta all'insoddisfazione risiede nella sincerità con cui si accetta la propria natura. Essa è a tratti incomprensibile, ma proprio per questo più interessante rispetto al vivere una vita qualunque, predeterminata da un collettivo che, al contrario degli astri, in cui, secondo gli antichi, sta scritta la storia di ciascuno di noi, non tiene conto delle nostre piccole atroci peculiarità.

Nome di battaglia è dunque una piacevole sfida ermeneutica per il lettore, ma un compito tutt'altro che facile per il suo traduttore, che si trova di fronte a una prosa intarsiata di espressioni molto connotate geograficamente e cronologicamente, trapunta di doppi sensi ambigui e di ironia enigmatica, per cui le scelte traduttive, ampiamente motivate nella nota al testo, si rivelano spesso coraggiose.

I due volumi di Almada editi da Urogallo permettono, dunque, ai lettori italiani di leggere o rileggere sotto una nuova luce uno degli artisti portoghesi più interessanti del Novecento. È una luce marina, un riverbero che ci arriva da alcuni di quelli che, per la fortuna di molti, hanno deciso di prendere il mare, invece di restare a terra. CLELIA BETTINI 